\title{
Study of the Electric Quadrupole Moments for some Scandium Isotopes Using Shell Model Calculations with Different Interactions
}

\author{
Ahmed H. Ali \\ Received 25/12/2017, Accepted 10/7/2018, Published 13/9/2018 \\ This work is licensed under a Creative Commons Attribution 4.0 International License.
}

\begin{abstract}
:
The electric quadrupole moments for some scandium isotopes $\left({ }^{41,43,44,45,46,47} \mathrm{Sc}\right)$ have been calculated using the shell model in the proton-neutron formalism. Excitations out of major shell model space were taken into account through a microscopic theory which is called core polarization effectives. The set of effective charges adopted in the theoretical calculations emerging about the core polarization effect. NushellX@MSU code was used to calculate one body density matrix (OBDM). The simple harmonic oscillator potential has been used to generate the single particle matrix elements. Our theoretical calculations for the quadrupole moments used the two types of effective interactions to obtain the best interaction compared with the experimental data. The theoretical results of the quadrupole moments for some scandium isotopes performed with FPD6 interaction and Bohr-Mottelson effective charge agree with experimental values.
\end{abstract}

Keywords: Bohr-Mottelson, Core Polarization, Effective Charges, Effective Charges, Harmonic Oscillator, Standard Effective charges.

\section{Introduction:}

The nuclear electric quadrupole moment, is considered as a probe for the deviation of nuclear charge from spherical symmetry which gives a useful measure of how the core is polarized especially if the valence nucleons are neutrons which do not directly participate to the electric quadrupole moment (1). Also, it gives information on the deformation of the charge distribution of the nucleus. The electromagnetic moments are one of the main probes to gain information about the nuclear structure throughout the entire nuclear chart (2). As the quadrupole moment provides a direct indication for changes in the nuclear shell structure along isotope chains, a proper determination of its size and sign is essential in both theoretical and experimental nuclear physics (3).

Richter et al.(4) used similar approaches to determine the 195 two-body matrix elements and the four single particle energies of the $f p$ shell. In one approach, the model-dependent potential method, a semiempirical interaction, was obtained by using one-boson exchange potential plus core polarization correction. The two interactions, i.e., FPD6 and FPM13, yield a good fit to the energy levels of nuclei in the lower part of the $f p$ shell.

The Kuo and Brown(5 )identified which matrix elements should be modified, while keeping the

Department of Medical Physics, College of Applied Sciences, University of Fallujah, Iraq.

E-mail: dr.ahmedphysics@uofallujah.edu.iq centroid of any two shells. This approach yielded the $K B 1$ interaction. Later, the $K B 3$ interaction was obtained, by doing mild changes in the $K B 1$ interaction to improve the spectroscopy of some nuclei at the beginning of the $f p$ shell. This interaction leads to fairly successful calculations in the $f p$ shell.

\section{Theory}

The electric and magnetic properties of a nuclear state, namely on what the static magnetic dipole and electric quadrupole moments can teach us about the nucleus as a system of independently moving particles in a central potential or as a system of collectively moving nucleons(6).

The one body electric multipole transition operator with multipolarity $J$ for a nucleon is given by (7)

$$
\widehat{O}_{J M}(\vec{r})_{k}=r_{k}^{J} Y_{J M}\left(\Omega_{K}\right)
$$

where $r_{k}^{J}$ is radial part of harmonic oscillator (HO) potential and $Y_{J M}$ is spherical part of HO potential.

The reduced single-particle matrix element of the transition operator given in Eq. (1) in spin space is given by

$\left\langle j^{\prime}|| \widehat{O}_{J t_{z}}|| j\right\rangle=e\left(t_{z}\right)\left\langle j^{\prime}|| Y_{J}|| j\right\rangle\left\langle n^{\prime} l^{\prime}\left|r^{J}\right| n l\right\rangle \ldots$

where; 
$e\left(t_{z}\right)=\frac{1+\tau_{z}(k)}{2} \quad$ is the electric charge of the $k$ nucleon, where $t_{z}=1 / 2$ for a proton and $t_{z}=-1 / 2$ for a neutron with

$\tau_{z}(k)=2 t_{z}(k), \tau_{z}|p\rangle=|p\rangle$ and $\tau_{z}|n\rangle=-|n\rangle \ldots$ (3)

,the reduce matrix element of the spherical harmonics part $Y_{J}$ is given by (7)

$$
\begin{aligned}
& \left\langle j^{\prime}|| Y_{J} \| j\right\rangle=(-1)^{j+1 / 2} \sqrt{\frac{(2 j+1)(2 J+1)\left(2 j^{\prime}+1\right)}{4 \pi}} \times \\
& \left(\begin{array}{ccc}
j^{\prime} & J & j \\
1 / 2 & 0 & -1 / 2
\end{array}\right) \times \frac{1}{2}\left[1+(-1)^{l+J+l^{\prime}}\right] \ldots
\end{aligned}
$$

and the radial part of the matrix element of a $\mathrm{HO}$ potential is: $\left\langle n^{\prime} l^{\prime}\left|r^{J}\right| n l\right\rangle=\int_{0}^{\infty} d r r^{J} r^{2} R_{n^{\prime} l^{\prime}}(r) R_{n l}(r) \quad \ldots$

Eq. (5) can be written as:

$\left\langle n^{\prime} l^{\prime}\left|r^{J}\right| n l\right\rangle=\int_{0}^{\infty} d r r^{\mu} R_{n^{\prime} l^{\prime}}(r) R_{n l}(r) \ldots$ (6) where $\mu=J+2$.

The radial integral Eq. (6) can solve analytically for a $\mathrm{HO}$ radial wave functions as (8).

$\int_{0}^{\infty} d r r^{\mu} R_{n^{\prime} l^{\prime}}(r) R_{n l}(r)=$

$\frac{2^{3}}{2^{2} 2} \sqrt{\left(n^{\prime}-1\right) !(n-1) !} \sqrt{\Gamma\left(n^{\prime}+l^{\prime}+\frac{1}{2}\right) \Gamma\left(n+l+\frac{1}{2}\right)}$

$$
\times \sum_{k^{\prime}=0}^{n^{\prime}-1} \sum_{k=0}^{n-1} \frac{(-1)^{k^{\prime}+k}}{\left(n^{\prime}-k^{\prime}-1\right) !(n-k-1) ! k^{\prime} ! k ! \Gamma\left(k^{\prime}+l^{\prime}+\frac{3}{2}\right) \Gamma\left(k+l+\frac{3}{2}\right)}
$$

$$
\times \frac{b^{2 m+1}}{b^{3+l^{\prime}+l+2 k^{\prime}+2 k}} \Gamma\left(m+\frac{1}{2}\right)
$$

where;

$$
\begin{aligned}
& m=\frac{1}{2}\left(\mu+l^{\prime}+l+2 k^{\prime}+2 k\right) \\
& \frac{b^{2 m+1}}{b^{3+l^{\prime}+l+2 k^{\prime}+2 k}}=b^{\mu-2} \\
& \Gamma\left(n+\frac{1}{2}\right)=\frac{(2 n-1) ! !}{2^{n}} \sqrt{\pi}
\end{aligned}
$$

The reduced electric matrix element between the initial and final nuclear states is given by (7):

$$
M(E J)=\left\langle J_{f}|| \sum_{k} e(k) \hat{O}_{J}(\vec{r})_{k}|| J_{i}\right\rangle \ldots
$$

$e(k)$ is the electric charge for the $k$ nucleon. Where $e(k)=0$ for neutron. The reduced electric matrix element can be written in terms of the proton and neutron contributions

$M(E J)=\sum_{t_{z}} e\left(t_{z}\right)\left\langle J_{f}\left\|\hat{O}_{J}\left(\vec{r}, t_{z}\right)\right\| J_{i}\right\rangle \ldots$ (9)

where $\left\langle J_{f}|| \widehat{O}_{J}\left(\vec{r}, t_{z}\right)|| J_{i}\right\rangle$ is the electric matrix element which is expressed as the sum of the products of the one-body density matrix (OBDM) times the single-particle matrix elements (7),

$\left\langle J_{f}|| \widehat{O}_{J}\left(\vec{r}, t_{z}\right) \mid J_{i}\right\rangle=$

$\sum_{j j} j^{O B D M}\left(J_{i}, J_{f}, J, t_{z}, j, j^{\prime}\right)\left\langle j^{\prime}|| \widehat{O}_{J}\left(\vec{r}, t_{z}\right)|| j\right\rangle \ldots$

with $j$ and $j$ label single-particle states for the shell model space.

The electric matrix element can be represented in terms of only the model space matrix elements by assigning effective charges $\left(e^{e f f}\left(t_{z}\right)\right)$,

$M(E J)=\sum_{t_{z}} e^{e f f}\left(t_{z}\right)\left\langle J_{f}\left\|\hat{O}_{2}\left(\vec{r}, t_{z}\right)\right\| J_{i}\right\rangle_{M S}$

They formulated an expression for the effective charges to explicitly include neutron excess via (9)

$e^{e f f}\left(t_{z}\right)=e\left(t_{z}\right)+e \delta e\left(t_{z}\right), \delta e\left(t_{z}\right)=Z / A-$ $0.32(N-Z) / A-2 t_{Z}[0.32-0.3(N-Z) / A]$ ...(12)
The electric quadrupole moment in a state $J=2 M=0>$ for $J_{i}=J_{f}$ is (7):

$Q(J=2)=\left(\begin{array}{ccc}J_{i} & J & J_{i} \\ -J_{i} & 0 & J_{i}\end{array}\right) \sqrt{\frac{16 \pi}{5}} M(E J)$

\section{Results and Discussion:}

The scandium isotopes $(Z=21)$ investigated in this study lie between the $Z, N=20$ and $Z, N=28$ shell closures. Shell-model calculations are performed by using the program NuShellX@MSU (10) to calculate OBDM with the $f p$ model space for $N \geq 20$, where ${ }^{40} \mathrm{Ca}$ nucleus is considered as the inert core for all isotopes under consideration and the other valence nucleons are moving in the $f p$ shell model space within $1 f_{7 / 2}, 2 p_{3 / 2}, 1 f_{5 / 2}$ and $2 p_{1 / 2}$ orbits. The $\mathrm{HO}$ is used to generate the single particle matrix elements of scandium isotopes. The present work in the $f p$-model space based two interactions: the $K B 3$ (Kuo and Brown) interaction for $f p$ - shell model space (5) and the FBD6 interaction for $f P$-shell model space (4). For $f p$ model space the 20 neutrons and protons are frozen in $s d$-shells. Effective charges are needed because of the polarization of the core which is not included in the model space. One set of effective charges, Bohr-Mottelson (B-M) (9) are calculated according to Eq. (12) and tabulated in Table 1. While, two set of effective charges, standard effective charges (ST) $e p=1.36 e$ and en $=0.45 e$ (11) were also calculated. Lastly, three sets of effective charges, conventional effective charges (Con) $e p=1.3 e$ and $e n=0.5 e$ (12) were computed.

The quadrupole moments are calculated for scandium isotopes with mass number $A=41,43$, 44, 45, 46, and 47. The matrix elements calculated with the shell model and $\mathrm{HO}$ wave functions also the radial wave functions for the single-particle matrix elements are calculated with the $\mathrm{HO}$ 
potential. The size parameters $b$ is calculated for each scandium isotope with mass number $A$ as:

$$
\begin{aligned}
b & =\sqrt{\frac{\hbar}{M_{P} \omega}}, \quad \text { where } \hbar \omega=45 A^{-1 / 3}-25 A^{-2 / 3} \\
(M p & =\text { mass of proton })(13) .
\end{aligned}
$$

Figure 1 and Table1 show the quadrupole

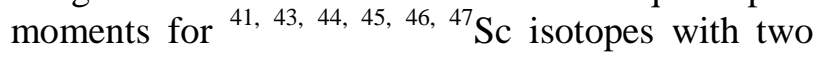
effective interactions and by using B-M (9) which are calculated according to Eq. (12) and tabulated in Table 1, for each isotope. The results of the quadrupole moments are displayed in Table 1 in comparison with the experimental values. The calculated quadrupole moment for ${ }^{41} \mathrm{Sc}(N=20)$ nucleus with FPD6 effective interaction and by using B-M effective charges for proton $e p=1.19 e$ and neutron $e n=0.85 e$ is $Q(\mathrm{~B}-\mathrm{M})=-13.5 \mathrm{efm}^{2}$, which agree with the measured values of Ref.(14). This value shows a large oblate deformation for $N=20$ although a number of neutrons are magically numbered and there were no theoretical results with using of $K B 3$ interaction due to the presence of one proton outside the inert core ${ }^{40} \mathrm{Ca}$. The calculated quadrupole moment for ${ }^{43} \mathrm{Sc}$ nucleus with $K B 3$ interaction, the FPD6 effective interaction and by using $\mathrm{B}-\mathrm{M}$ effective charges for proton $e p=1.17 e$ and neutron en=0.79e are $Q(\mathrm{~B}-\mathrm{M})=-20.8,-20.5$ $e f m^{2}$, which are overestimates of the measured values of Ref. (14). Furthermore, calculations of the quadrupole moments of ${ }^{45} \mathrm{Sc}$ nucleus with $K B 3$ interaction, FPD6 effective interaction and by using $\mathrm{B}-\mathrm{M}$ effective charges for proton $e p=1.15 e$ and neutron $e n=0.75 e$, respectively, $Q(\mathrm{~B}-\mathrm{M})=-23.16$, $23.6 \mathrm{efm}^{2}$. Calculations of quadrupole moment for ${ }^{47} \mathrm{Sc}$ nucleus with KB3 interaction, the FPD6 effective interaction and by using B-M effective charges for proton $e p=1.12 e$ and neutron $e n=0.7 e$, $Q(\mathrm{~B}-\mathrm{M})=-21.88,-22.59 \mathrm{efm}^{2}$ which are close to the measured values of Ref. (14) these value shows an oblate deformation. Calculations of the quadrupole moments for ${ }^{44} \mathrm{Sc}$ nucleus with FPD6 effective interaction and by using B-M effective charges are overestimating the measured values while by using $K B 3$ interaction are close the measure values. Calculations of the quadrupole moments for ${ }^{46} \mathrm{Sc}$ are underestimated with the measured values of Ref. (14) where positive parity is. The result of the quadrupole moment for ${ }^{46} \mathrm{Sc}$ indicates a small prolate deformation of this isotope which needs further research.

Table 1. Quadrupole moments in units efm2 of calculated with HO potential for Scandium (Sc) isotopes $(\mathrm{Z}=21)$. Experimental the quadrupole moments are taken from Ref. (14). Quadrupole moments calculated with two effective interactions, the first $K B 3$ interaction (5) and the second FBD6 interaction (4) and use effective charges of B-M (9) are presented.

\begin{tabular}{cccccccc}
\hline $\boldsymbol{A}, \boldsymbol{N}_{2 \mathbf{1}} \mathbf{S c}$ & $\boldsymbol{J}_{\boldsymbol{i}}^{\boldsymbol{\pi}}$ & $\boldsymbol{b}(\boldsymbol{f m})$ & $\boldsymbol{e}_{\boldsymbol{p}}$ & $\boldsymbol{e}_{\boldsymbol{n}}$ & $\begin{array}{c}\boldsymbol{Q}_{\text {theo }} \boldsymbol{B}-\boldsymbol{M} \\
\boldsymbol{K B 3} \text {-int }\end{array}$ & $\begin{array}{c}\boldsymbol{Q}_{\text {theo. }} \boldsymbol{B} \text { - } \boldsymbol{M} \\
\boldsymbol{F P D 6} \text {-int }\end{array}$ & $\boldsymbol{Q}_{\text {exp. }}\left(\boldsymbol{e f m}^{2}\right)$ \\
\hline 41,20 & $7 / 2^{-}$ & 1.947 & 1.19 & 0.85 & & -13.5 & $-14.5 \pm 3$ \\
43,22 & $7 / 2^{-}$ & 1.96 & 1.17 & 0.79 & -20.8 & -20.5 & $-27 \pm 5$ \\
44,23 & $2^{+}$ & 1.966 & 1.16 & 0.77 & 7.56 & 10.56 & $1 \pm 5$ \\
45,24 & $7 / 2^{-}$ & 1.972 & 1.15 & 0.75 & -23.16 & -23.6 & $-22 \pm 2$ \\
46,25 & $4^{+}$ & 1.978 & 1.13 & 0.72 & 2 & 4.07 & $12 \pm 2$ \\
47,26 & $7 / 2^{-}$ & 1.983 & 1.12 & 0.70 & -21.88 & -22.59 & $-22 \pm 3$ \\
\hline
\end{tabular}

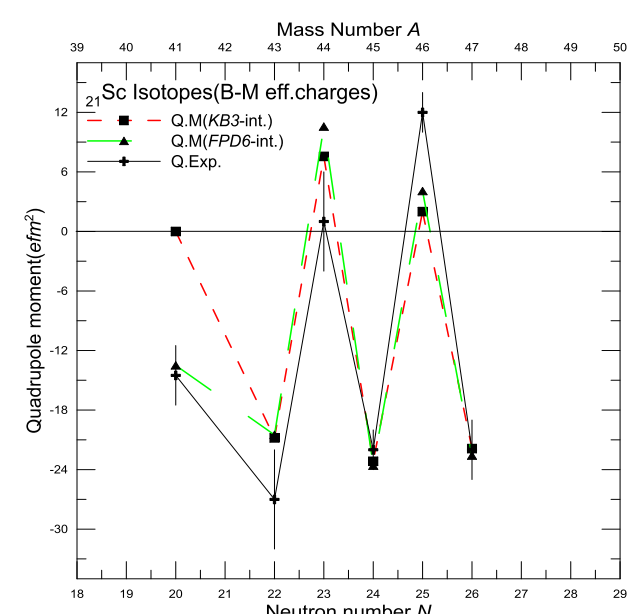

Figure1. Calculations of the quadrupole moments by using two effective interaction and Bohr-Mottelson (B-M) effective charges (9) for Sc isotopes. Experimental values are taken from Ref. (14).
Figure 2 and Table 2 show the quadrupole

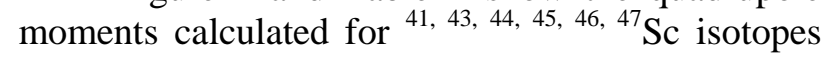
with two effective interactions, the first $K B 3$ interaction for $f p$ - shell model space (5) and the second FBD6 interaction for $f P$-shell model space (4) and by using the standard effective charges ST for proton $e p=1.36 e$ and neutron $e n=0.45 e(11)$, respectively. The results of the quadrupole moments are displayed in Table 2 in comparison with the experimental values. The calculated quadrupole moment for ${ }^{41} \mathrm{Sc}$ nucleus with FPD6 effective interaction and using standard effective charges $Q(\mathrm{ST})=-15.46 \mathrm{efm}^{2}$, agree with the measured values of Ref.(14).

This value shows a large oblate deformation for $N=20$ although a number of neutrons are magically numbered. The calculated the quadrupole moment for ${ }^{43} \mathrm{Sc}$ nucleus with $K B 3$ interaction and 
FPD6 effective interaction and using ST for proton $e p=1.36 e$ and neutron $e n=0.45 e$ (11), respectively are $Q(\mathrm{ST})=-17.8,-17.7 \mathrm{efm}^{2}$, overestimate the measured values of Ref. (14). Also, calculations of the quadrupole moments for ${ }^{45} \mathrm{Sc}$ nucleus with $K B 3$ interaction, FPD6 effective interaction and by using ST, $Q(\mathrm{ST})=-20.2,-20.7 \mathrm{efm}^{2}$, which agree with measured values. Calculations of the quadrupole moments for ${ }^{47} \mathrm{Sc}$ nucleus with $K B 3$ interaction, FPD6 effective interaction and by using standard effective charges for proton $e p=1.36 e$ and neutron $e n=0.45 e, Q(\mathrm{ST})=-20.56,-20.15 \mathrm{efm}^{2}$, which agree with the measured values of Ref.(14) these values shows an oblate deformation because one proton in $f p$-shell had negative parity. Calculations of the quadrupole moments for ${ }^{44} \mathrm{Sc}$ nucleus with FPD6 effective interaction and by using ST effective charges are overestimating the measured values while by using $K B 3$ interaction are very close to the measure values. Calculations of the quadrupole moments for ${ }^{46} \mathrm{Sc}$ underestimate the measured values of Ref. (14) where positive parity is and the result of quadrupole moment for ${ }^{46} \mathrm{Sc}$ indicates a small prolate deformation of this isotope which needs further research.

Table 2: Quadrupole moments in units $\mathrm{efm}^{2}$ of calculated with $\mathrm{HO}$ potential for Scandium isotopes $(\mathrm{Z}=21)$. Experimental quadrupole moments are taken from Ref. (14). Quadrupole moments calculated with two effective interactions, the first $K B 3$ interaction (5) and the second FBD6 interaction (4) and use standard effective charges for proton and neutron $1.36,0.45 e(11)$, respectively are presented.

\begin{tabular}{cccccccc}
\hline $\begin{array}{c}\boldsymbol{A}, \boldsymbol{N} \\
{ }_{21} \mathbf{S c}\end{array}$ & $\boldsymbol{J}_{\boldsymbol{i}}^{\boldsymbol{\pi}}$ & $\boldsymbol{b}(\boldsymbol{f m})$ & $\boldsymbol{e}_{\boldsymbol{p}}$ & $\boldsymbol{e}_{\boldsymbol{n}}$ & $\begin{array}{c}\boldsymbol{Q}_{\text {theo. }} \\
\boldsymbol{S T}\end{array}$ & $\begin{array}{c}\boldsymbol{Q}_{\text {theo. }} \\
\boldsymbol{S T} \\
\boldsymbol{F P D 6 - i n t}\end{array}$ & $\begin{array}{c}\boldsymbol{Q}_{\boldsymbol{e x p}} \\
\left(\boldsymbol{e f m}_{\mathbf{2}}\right.\end{array}$ \\
\hline 41,20 & & & & & & -15.46 & $-14.5 \pm 3$ \\
43,22 & $7 / 2^{-}$ & 1.947 & 1.36 & 0.45 & & -17.7 & $-27 . \pm 5$ \\
44,23 & $2^{+}$ & 1.96 & 1.36 & 0.45 & -17.8 & 8.7 & $1.0 \pm 5$ \\
45,24 & $7 / 2^{-}$ & 1.972 & 1.36 & 0.45 & 6.2 & -20.7 & $-22 . \pm 2$ \\
46,24 & $4^{+}$ & 1.978 & 1.36 & 0.45 & -20.2 & 1.7 & $12 \pm 2$ \\
47,26 & $7 / 2^{-}$ & 1.983 & 1.36 & 0.45 & 0.21 & -21.15 & $-22 \pm 3$ \\
\hline
\end{tabular}

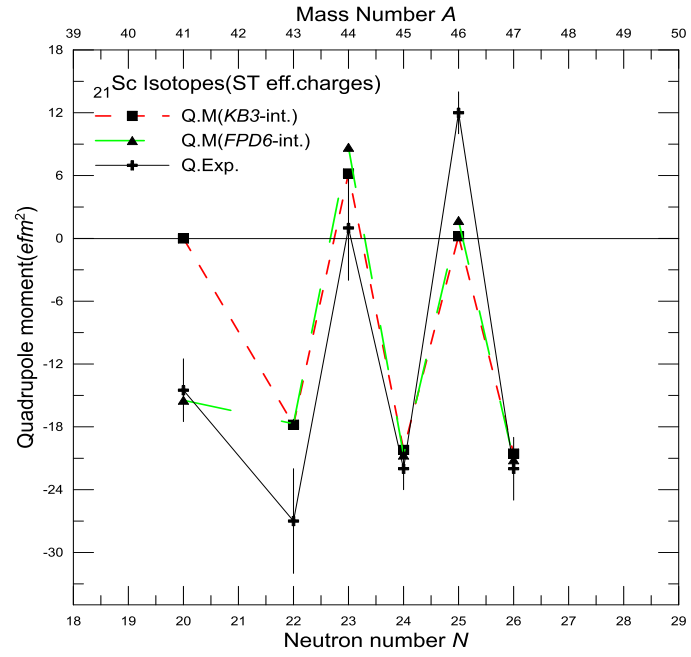

Figure 2. Calculations of the quadrupole moments by using two effective interaction and by using the standard effective charges for proton and neutron $1.36,0.45 e(11)$, respectively for Sc isotopes. Experimental values are taken from Ref. (14).

Figure 3 and Table 3 show the quadrupole moments are calculated for ${ }^{41}, 43,44,45,46,{ }^{47} \mathrm{Sc}$ isotopes with two effective interactions, the first $K B 3$ interaction for $f p$ - shell model space (5) and the second FBD6 interaction for $f P$-shell model space (4) and use convention effective charges (Con) for proton $e p=1.3 e$ and neutron $e n=0.5 e$ (12), are presented. The results of the quadrupole moments are displayed in Table 3 in comparison with the experimental values. The calculated quadrupole moment for ${ }^{41} \mathrm{Sc}$ nucleus with FPD6 effective interaction and by using convention effective charges $Q(\mathrm{Con})=-14.78 \mathrm{efm}^{2}$ agrees with the measured values of Ref. (14), and our values shows an oblate deformation. The calculated quadrupole moment for ${ }^{43} \mathrm{Sc}$ nucleus by using $K B 3$ interaction and FPD6 effective interaction and by using convention effective charges for proton $e p=$ $1.3 e$ and neutron $e n=0.5 e$ (12), respectively is $Q($ Con $)=-18,-17.8 \mathrm{efm}^{2}$, which are overestimates the measured values of Ref.(14). Also, calculations of the quadrupole moments for ${ }^{45} \mathrm{Sc}$ nucleus by using KB3 interaction and FPD6 effective interaction and by using convention effective charges for proton $e p=1.3 e$ and neutron $e n=0.5 e$, $Q($ Con $)=-20.5,-20.5 \mathrm{efm}^{2}$ which agree with the measured values of Ref.(14). Calculations of quadrupole moment for ${ }^{47} \mathrm{Sc}$ nucleus by using $K B 3$ interaction and FPD6 effective interaction and by using convention effective charges is $Q(\mathrm{Con})=$ $-20.69,-21.3 \mathrm{efm}^{2}$ which agree with the measured values of Ref.(14), and show an oblate deformation. Calculations of the quadrupole moments for ${ }^{44} \mathrm{Sc}$ nucleus with FPD6 effective interaction and by using ST effective charges are overestimating the measured values while by using $K B 3$ interaction are close to the measure values. Calculations the quadrupole moments for ${ }^{46} \mathrm{Sc}$ underestimate the 
measured values of Ref. (14) where positive parity is. The result of quadrupole moment for ${ }^{46} \mathrm{Sc}$ indicates a small prolate deformation of this isotope which needs further research.

Table 3: Quadrupole moments in units $\mathrm{efm}^{2}$ of calculated with HO potential for Scandium (Sc) isotopes $(Z=21)$. Experimental quadrupole moments are taken from Ref. (14). Quadrupole moments calculated with two effective interactions, the first $K B 3$ interaction (5) and the second $F B D 6$ interaction (4) and use convention effective charges proton and neutron $1.3 e, 0.5 e(12)$, respectively are presented.

\begin{tabular}{cccccccc}
\hline $\boldsymbol{A}, \boldsymbol{N}_{\mathbf{2 1}} \mathbf{S c}$ & $\boldsymbol{J}_{\boldsymbol{i}}^{\boldsymbol{\pi}}$ & $\boldsymbol{b}(\boldsymbol{f m})$ & $\boldsymbol{e p = 1 . 3}$ & $\boldsymbol{e n = 0 . 5}$ & $\begin{array}{c}\boldsymbol{Q}_{\text {theo }} \text { con } \\
\boldsymbol{k b 3 - i n t}\end{array}$ & $\begin{array}{c}\boldsymbol{Q}_{\text {theo }} \text {. con } \\
\text { fpd6-int }\end{array}$ & $\boldsymbol{Q}_{\text {exp. }}$ \\
\hline 41,20 & $7 / 2^{-}$ & 1.947 & 1.3 & 0.5 & & -14.78 & $-14.5 \pm 3$ \\
43,22 & $7 / 2^{-}$ & 1.96 & 1.3 & 0.5 & -18 & -17.8 & $-27 . \pm 5$ \\
44,23 & $2^{+}$ & 1.966 & 1.3 & 0.5 & 6.39 & 8.9 & $1.0 \pm 5$ \\
45,24 & $7 / 2^{-}$ & 1.972 & 1.3 & 0.5 & -20.5 & -20.5 & $-22 . \pm 2$ \\
46,25 & $4^{+}$ & 1.978 & 1.3 & 0.5 & 0.57 & 2.16 & $12 \pm 2$ \\
47,26 & $7 / 2^{-}$ & 1.983 & 1.3 & 0.5 & -20.69 & -21.3 & $-22 \pm 3$ \\
\hline
\end{tabular}

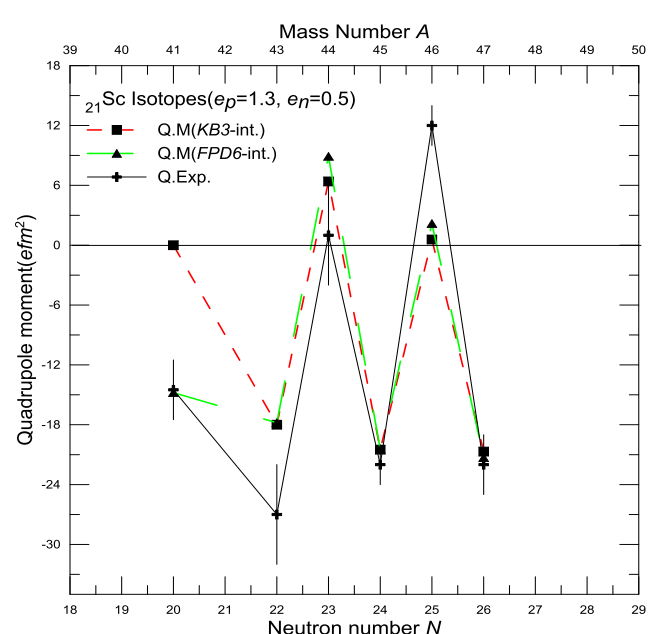

Figure 3. Calculations of the quadrupole moments by using two effective interaction and the convention effective charges proton and neutron $1.3,0.5 e(12)$, respectively for $\mathrm{Sc}$ isotopes. Experimental values are taken from Ref. (14).

\section{Conclusions:}

The results that obtained from this work have been deduced by using the shell model calculations, which were successful in describing the quadrupole moment when taking the core polarization effects into account. Calculations of quadrupole moments with FPD6 interaction and by using B-M effective charges are better than using the KB3 interaction. Calculations of quadrupole moments with FPD6 interaction and by using ST effective charges are better than using the KB3 interaction. Calculations of the quadrupole moments with FPD6 interaction and by using convention effective charges are better than using the $K B 3$ interaction. Finally, the calculations of the quadrupole moments with FPD6 interaction and by using B-M effective charge are better than those of ST effective charges and conventional effective charges.

\section{Reference:}

1. Raad A, Ahmed H. Microscopic Effective Charges and Quadrupole Moments of $S d$-shell and $S d$-Pf Cross-Shell Nuclei with $Z \geq 13$. Iraqi J. Phys. 2016; 14(30): 136-149.

2. Raad A, Zaheda A, Noori S. Quadrupole Moment of ${ }^{14}$ B Exotic Nucleus. Iraqi J. Phys. 2014: 12-50.

3. De Rydt M, Depuydt M, Neyens G. Evaluation of the Ground-State Quadrupole Moments of the $\pi(s d)$ Nuclei. Atomic Data and Nuclear Data Tables. 2013; 99: 391-415.

4. Werner A, Johan VD, Julies R, Alex B. New Effective Interactions for the Oflp Shell. Nucl. Phys.1991; A523:325.

5. Ifredo $\mathrm{P}$, Andreas Z. Theoretical Spectroscopy and $f p$ Shell. Phys. Rep.1981; 70: 235-314.

6. Ban S. The Nuclear Structure for Exotic NeutronRich of ${ }^{42,43,45,47}$ K Nuclei. BSJ. 2016; 13.

7. Brussard P, Glaudemans P. Shell-Model Applications in Nuclear Spectroscopy. North Holland. Amesterdam. 1977; 452.

8. Raad A, Ali A, Ahmed H. Calculations of the Quadrupole Moments for Some Nitrogen Isotopes in $p$ and $p s d$ Shell Model Spaces Using Different Effective Charges. Iraqi J. Sci. 2017; 58(2): 878-883.

9. Raad A, Ahmed H. Microscopic Calculations of Effective Charges and Quadrupole Transition Rates in $\mathrm{Si}, \mathrm{S}$ and Ar Isotopes. Iraqi J. Sci. 2016; 57(3): 999-2013.

10. Alex B, Rae WD. The Shell-Model Code NuShellX@MSU. Nucl. Data Sheets; 2014; 120: 115.

11. Werner A, Mkhize S, Alex B. Sd-Shell Observables for the USDA and USDB Hamiltonians. Phys. Rev. C. 2008; 78; 064302.

12. Alex B. The Nuclear Shell Model Towards the Drip Lines. Prog. Part. Nucl. Phys. 2001; 47: 517-599.

13. Alex B, Raad A, Bryan H. Electric Quadrupole and Hexadecupole Nuclear Excitations from the Perspectives of Electron Scattering and Modern Shell-Model Theory. Phys. Rep. 1983; 101: 313. 
14. Stone J. Table of Nuclear Electric Quadrupole

2016; $112: 28$.

Moments. Atomic Data and Nuclear Data Tables;

\section{بأستعمال حسابات نموذج القشرة دراسة عزوم رباعية القطب في بعض نظائر سكانديوم مع تفاعلات مختلفة}

\section{احمد حسين علي}

قسم الفيزياء الطبية ، كلية العلوم التطبيقية ، جامعة الفلوجة، العراق.

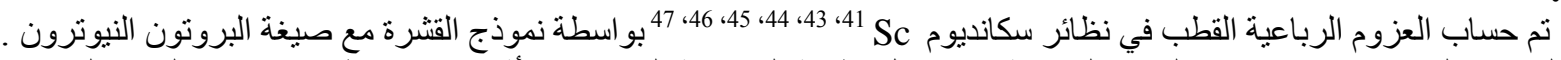

الخلاصة:

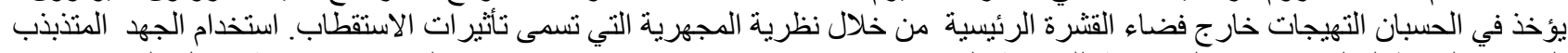

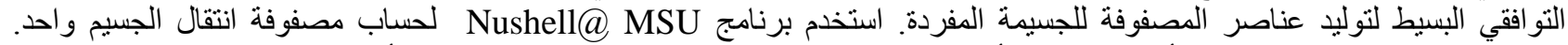

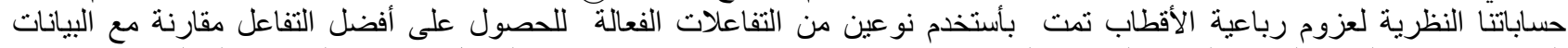

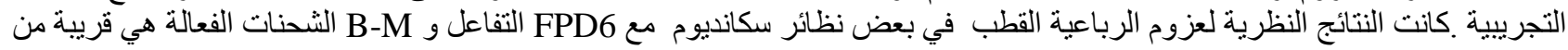
البيانات التجريبية .

الكلمات المفتاحية: شحنات بور موتلسن الفعالة، ستقطاب القلب، شحنات فعالة، المتذبذب التو افقي، شحنات فعالة قياسية. 